Archive for

Organic Chemistry

Arkivoc 2020, part vi, 94-104

\title{
Synthesis of novel NHC-Rh complexes with anti-tumor activity against MCF-7 human breast cancer cells
}

\author{
Xiong Zhao, ${ }^{1,}{ }^{*}$ Lu Shi, ${ }^{2}$ Weiping $\mathrm{He},{ }^{2}$ Fan Yang, ${ }^{2}$ and Jie $\mathrm{Li}^{2,}$ \\ ${ }^{1}$ Department of Burn \& Plastic Surgery, Children's Hospital, Zhejiang University School of Medicine, 3333 \\ Binsheng Road, Hangzhou 310052, China \\ ${ }^{2}$ Department of Pharmacy, School of Medicine, Zhejiang University City College, No. 48, Huzhou Road, \\ Hangzhou 310015, China
}

Email: 6196001@zju.edu.cn lijie@zucc.edu.cn

Received 12-15-2019

Accepted 04-27-2020

Published on line $04-29-2020$

\section{Abstract}

A series of novel $\mathrm{N}$-heterocyclic carbene rhodium complexes (NHC-Rh), containing benzimidazole framework, were prepared smoothly by a five-step route. Their structures were elucidated based on spectral techniques, including NMR and MS spectrometry. Along with several NHC-Rh complexes we prepared before, the compounds prepared herein were evaluated for their cytotoxicities against MCF human breast cancer cells and several of them showed strong activities with $\mathrm{IC}_{50}$ values ranging from 0.38 to $1.18 \mu \mathrm{M}$, which were better than the positive control paclitaxel.

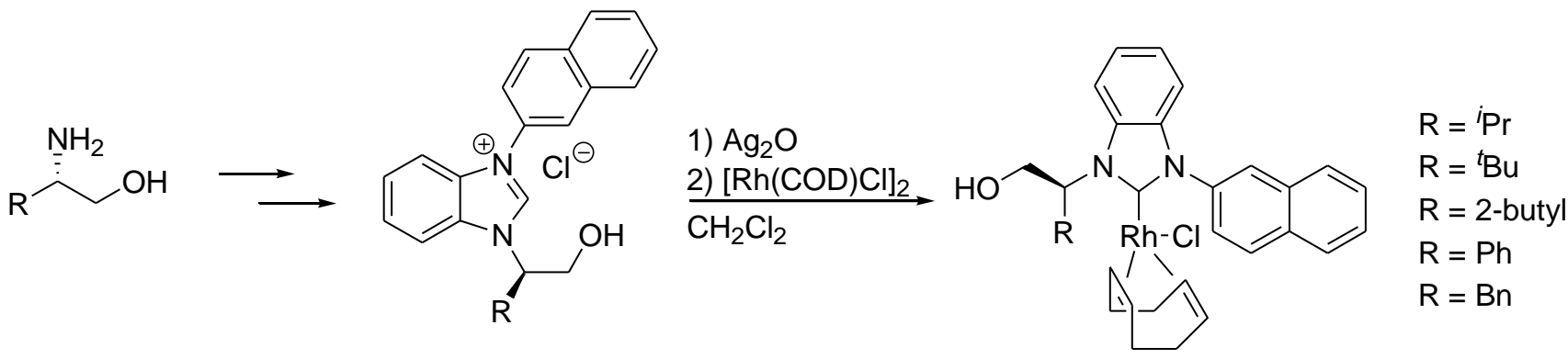

Keywords: N-heterocyclic carbene; benzimidazolium; cytotoxic activity; MCF-7 cell lines 


\section{Introduction}

In 1960s, Öfele and Wanzlick reported the first example of N-heterocyclic carbenes (NHCs). ${ }^{1-2}$ However, little attention was paid to these kind of ligands until Arduengo and co-workers prepared the first isolable carbene in $1991{ }^{3} \mathrm{NHCs}$ are famous for their powerful $\sigma$-donating ability due to the adjacent nitrogen atoms around carbene $\mathrm{C}$ atom. Therefore, employment of $\mathrm{N}$-heterocyclic carbenes (NHCs) as ligands to generate coordination compounds have attracted considerable attention in homogeneous metal catalysis. ${ }^{4-13}$ Due to their excellent $\sigma$-donating ability, increasing NHCs appeared as ligands to construct complexes in metal medicine. ${ }^{14}$ In this respect, NHC ligands may substantially stabilize metal complexes to resist degradation under physiological conditions. Notably, the modification at ${ }^{1} \mathrm{~N}$ and ${ }^{3} \mathrm{~N}$ positions of $\mathrm{NHC}$ ligands is facile and a variety of functional groups could be introduced to these ligands to increase the selectivity to biomolecules. ${ }^{15}$ Since Berners-Price reported the anticancer properties of $\mathrm{Au}(\mathrm{I})$-NHC complexes in 2004, ${ }^{16}$ considerable efforts have been dedicated to the investigation of metal NHC complexes, such as $\mathrm{Au}, \mathrm{Ag}, \mathrm{Pd}, \mathrm{Pt}, \mathrm{Cu}, \mathrm{Ru}$, and $\mathrm{Ir}$, as antineoplastic agents. ${ }^{17-22}$ In combination of our interest in the development of novel NHCs ligands ${ }^{23-28}$ and our experience with benzimidazole carbenes, ${ }^{29-31}$ we herein report a new route for the synthesis NHC-Rh complexes with benzimidazole backbone using simple 1,2-dibromobenzene as starting material. Considering the promising potential of rhodium complexes as therapeutic molecules, ${ }^{32-39}$ these new complexes were evaluated for cytotoxicity against MCF-7 human breast cancer cells and most of the synthesized complexes showed excellent activities.

\section{Results and Discussion}

As shown (Scheme 1), the novel NHC-Rh complexes were synthesized by a five-step route. To protect the hydroxyl group, the commercial available amino alcohols $1 \mathrm{a}-1 \mathrm{e}$ were firstly treated with $t$-butyldimethylsilyl chloride, affording the silylanization products $\mathbf{2 a - 2 e}$ in good yields $(74-88 \%)$. The corresponding products were then underwent a Buchwald-Hartwig coupling with 1,2-dibromobenzene, giving the monosubstituted products $3 \mathbf{a}-\mathbf{3 e}$ in $68-93 \%$ yields. A second Buchwald-Hartwig coupling was then conducted with 2naphthylamine, furnishing the differentially substituted diamines $4 a-4 e$ in excellent yields (88-98\%). Finally, the benzimidazolium salts $\mathbf{5 a - 5 e}$ were prepared smoothly by treatment of the corresponding diamines with concentrated hydrochloric acid in $\mathrm{CH}(\mathrm{OEt})_{3}$, giving the products in $80-87 \%$ yields. Interestingly, the TBS group was deprotected simultaneously in this cyclization step.

With benzimidazolium precursors in hand, the NHC-Rh complexes 6a-6e were prepared without difficulty using a transmetalation method developed by Lin and co-workers, ${ }^{40}$ and the procedure was depicted as follow. The benzimidazolium salts $\mathbf{5 a}-\mathbf{5 e}$ were treated with $\mathrm{Ag}_{2} \mathrm{O}$ in $\mathrm{CH}_{2} \mathrm{Cl}_{2}$ at room temperature for 2 hours in the darkness. The resulting reaction mixture was used directly with the addition of $[\mathrm{Rh}(\mathrm{COD}) \mathrm{Cl}]_{2}$, and the NHC-Rh complexes 6a-6e were constructed upon workup. The target product is air stable and could be purified by chromatography on silica gel without decomposition. 


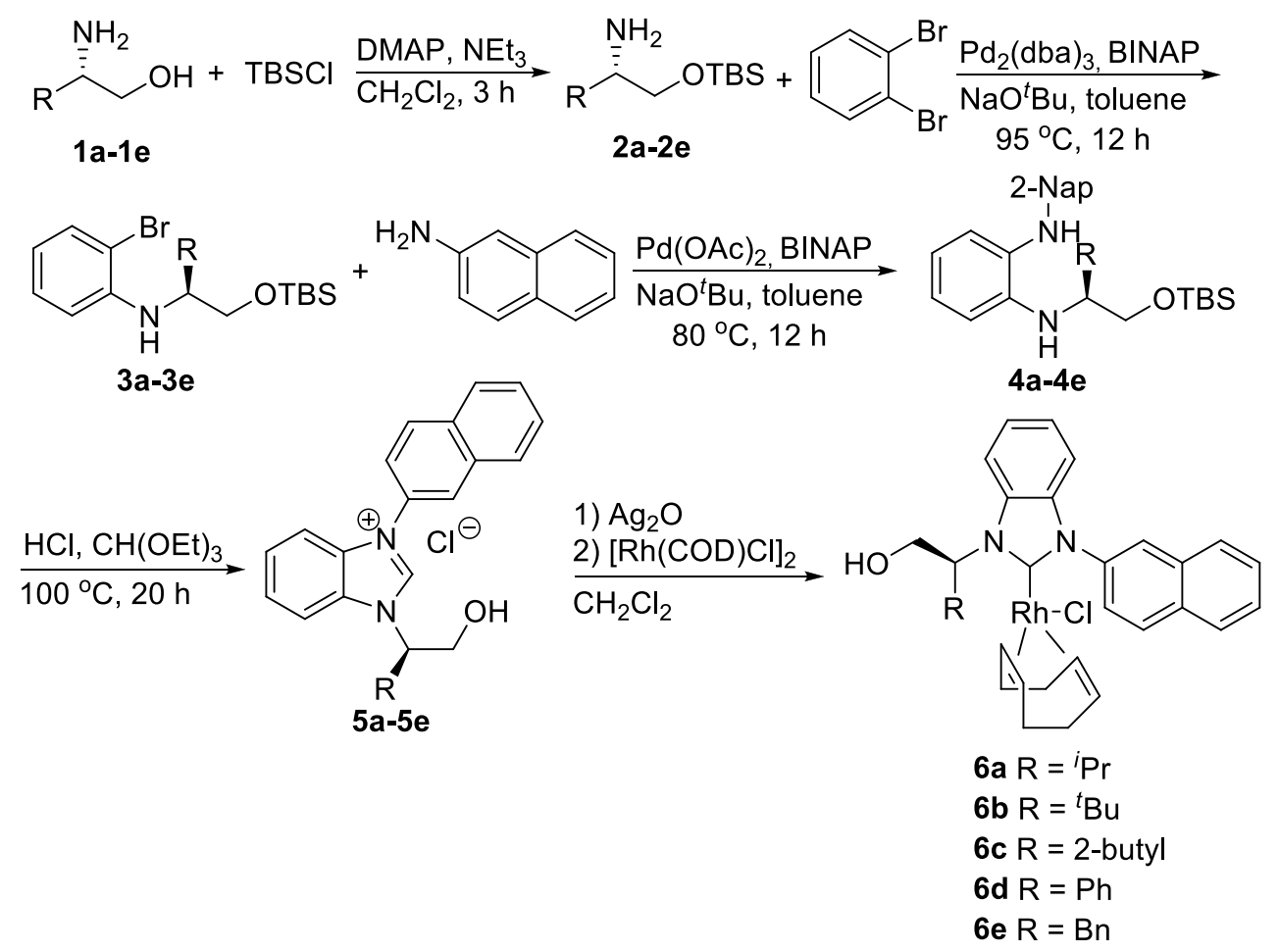

Scheme 1. Synthesis of new NHC-Rh complexes 6a-6e.

In our previous work, we have prepared a series of $\mathrm{NHC}-\mathrm{Rh}$ complexes $\mathbf{7 a} \mathbf{- 7} \mathbf{g}$ with benzimidazole backbone through a different method. ${ }^{29,31}$ The NHC-Rh complexes prepared herein distinguish from compounds $\mathbf{7 a - 7 g}$ for incorporating hydroxyl group in one of the $\mathrm{N}$-substituents, which may stabilize the metal center by hydrogen bond. Using paclitaxel as a positive control, the NHC-Rh complexes 7a-7g (Figure 1), along with the novel complexes $6 \mathbf{a}-6 \mathrm{e}$ in this study, were subjected to evaluate cytotoxicity against MCF-7 human breast cancer cells. As shown in Table 1, compounds 6a, 6b, 6c, 6e, 7c, 7d, and 7g exhibited strong activities with $\mathrm{IC}_{50}$ values ranging from 0.385 to $1.18 \mu \mathrm{M}$, which is better than paclitaxel. The promising results indicated the following primary structure activity relationship: (1) introduction of hydroxyl group at $N$ substituents is beneficial for the antitumor activity of these NHC-Rh complexes. (2) the alkyl groups at C-2' is essential to maintain the biological activity. Overall, the results presented herein may contribute a growing list of bioactive rhodium complexes and provide lead compounds for development of more effective antineoplastic agents. 


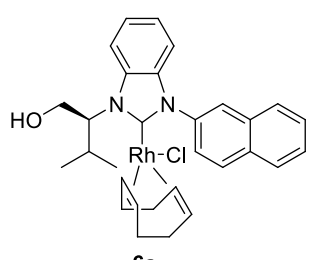

$6 a$

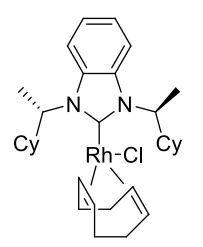

$7 \mathrm{a}$

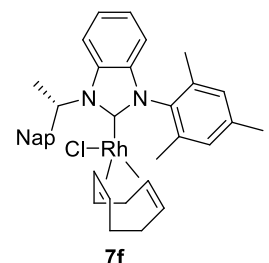

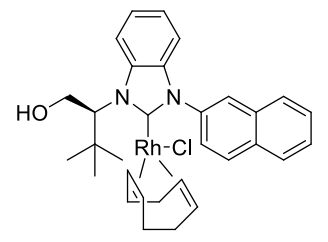

$6 b$

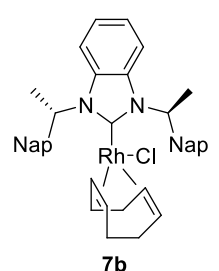

$7 \mathbf{b}$

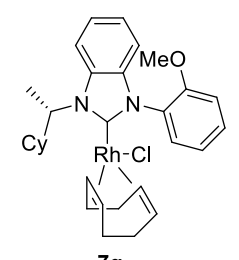

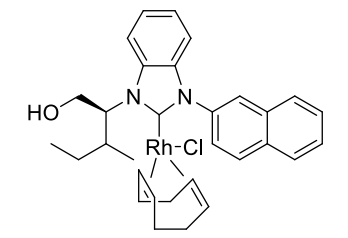

$6 c$

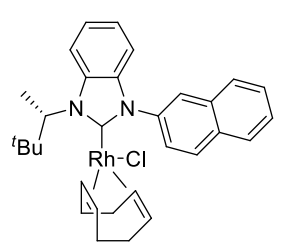

7c

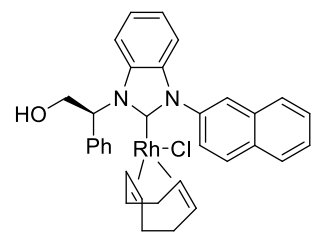

$6 \mathrm{~d}$

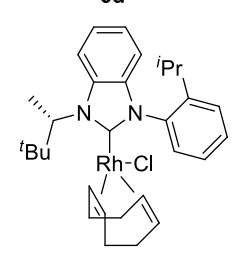

7d

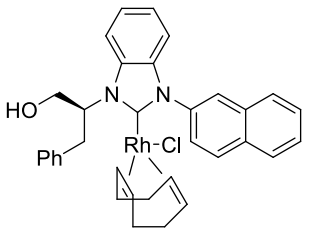

$6 \mathrm{e}$

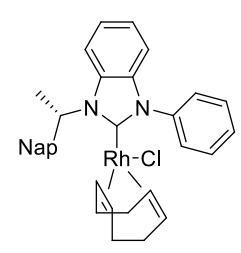

$7 \mathrm{e}$

Figure 1. Structure of NHC-Rh complexes $6 a-6 e$ and $7 a-7 g$.

Table 1. IC 50 values of NHC-Rh complexes against MCF-7 human breast cancer cell

\begin{tabular}{ccc}
\hline Entry & Compound & $\mathrm{IC}_{50}(\mu \mathrm{M})$ \\
\hline 1 & $\mathbf{6 a}$ & 0.38 \\
2 & $\mathbf{6 b}$ & 0.45 \\
3 & $\mathbf{6 c}$ & 0.72 \\
4 & $\mathbf{6 d}$ & $>10$ \\
5 & $\mathbf{6 e}$ & 1.02 \\
6 & $\mathbf{7 a}$ & $>10$ \\
7 & $\mathbf{7 b}$ & $>10$ \\
8 & $\mathbf{7 c}$ & 0.54 \\
9 & $\mathbf{7 d}$ & 1.18 \\
10 & $\mathbf{7 e}$ & $>10$ \\
11 & $\mathbf{7 f}$ & $>10$ \\
12 & $\mathbf{7 g}$ & 0.61 \\
13 & Paclitaxel & 1.38 \\
\hline
\end{tabular}

\section{Conclusions}

In summary, we described herein a facile route for the synthesis of novel NHC-Rh compexes with benzimidazole framework. This approach enables the preparation of a series of complexes $6 \mathbf{a}-6 \mathrm{e}$ bearing similar backbone but incorporating different $\mathrm{N}$-substituents. Comparing to our reported complexes $\mathbf{7 a - 7 g}$, the newly complexes which incorporating hydroxyl group showed better result in the test for their biological activity against MCF-7 human cancer cell lines. This study provides the promising compounds for further drug 
candidate development. Further application of these Rh-complexes in asymmetric catalysis are currently underway and will be reported in due course.

\section{Experimental Section}

General. ${ }^{1} \mathrm{H}$ - and ${ }^{13} \mathrm{C}-\mathrm{NMR}$ spectra were obtained on Bruker AVANCE III $500 \mathrm{MHz}$ and $600 \mathrm{MHz}$ spectrometers (Bruker Co., Billerica, MA, USA) with TMS as the internal standard; MS spectra were measured on a Finnigan LCQDECA XP instrument and an Agilent Q-TOF 1290 LC/6224 MS system (Santa Clara, CA, USA); silica gel GF 254 and H (10-40 mm, Qingdao Marine Chemical Factory, Qingdao, China) were used for TLC and CC. Unless otherwise noted, all reactions were carried out under an atmosphere of nitrogen.

Procedure for the synthesis of compounds 2a-2e. A solution of $t$-butylchlorodimethylsilane (TBSCl) (1.5 g, 10 $\mathrm{mmol}$ ) in $\mathrm{CH}_{2} \mathrm{Cl}_{2}(50 \mathrm{~mL})$ was dropped to a stirred solution of (S)- valinol (1a) (1.03 g, $\left.10 \mathrm{mmol}\right), \mathrm{Et}_{3} \mathrm{~N}(2.02 \mathrm{~g}, 20$ mmol), and 4-(dimethylamino)pyridine (DMAP) $(0.244 \mathrm{~g}, 2.0 \mathrm{mmol})$ in $\mathrm{CH}_{2} \mathrm{Cl}_{2}(16 \mathrm{~mL})$. After stirring at room temperature for $18 \mathrm{~h}$, the mixture was poured into water, and extracted with $\mathrm{CH}_{2} \mathrm{Cl}_{2}(30 \mathrm{~mL} \times 3)$. The solvents were removed under reduced pressure and the residue was purified by column chromatography $\left(\mathrm{CH}_{2} \mathrm{Cl}_{2}: \mathrm{MeOH}=70: 1\right)$ to give the product $2 \mathrm{a}$ as colorless oil $\left(74 \%\right.$ yield). ${ }^{1} \mathrm{H} \mathrm{NMR}\left(500 \mathrm{MHz}, \mathrm{CDCl}_{3}\right) \delta: 3.64$ (dd, J 9.8, 4.0 Hz, 1H), $3.40-3.37(\mathrm{~m}, 1 \mathrm{H}), 2.59-2.56(\mathrm{~m}, 1 \mathrm{H}), 0.92(\mathrm{~d}, \mathrm{~J} 4.4,6 \mathrm{H}), 0.90(\mathrm{~s}, 9 \mathrm{H}), 0.06(\mathrm{~s}, 6 \mathrm{H})$. The ${ }^{1} \mathrm{H}$ NMR data of $2 a$ was identical to those reported in the literature. ${ }^{41}$

Analogous compounds $\mathbf{2} \mathbf{b}-\mathbf{2 e}$ were prepared according to the similar procedure for $\mathbf{2 a}$. $\mathbf{2 b}$ : $75 \%$ yield; ${ }^{1} \mathrm{H}$ NMR $\left(500 \mathrm{MHz}, \mathrm{CDCl}_{3}\right) \delta: 3.76(\mathrm{dd}, J 9.8,3.2 \mathrm{~Hz}, 1 \mathrm{H}), 3.37-3.34(\mathrm{~m}, 1 \mathrm{H}), 2.57(\mathrm{dd}, J 8.8,3.2 \mathrm{~Hz}, 1 \mathrm{H}), 0.92(\mathrm{~s}, 9 \mathrm{H})$, $0.91(\mathrm{~s}, 9 \mathrm{H}), 0.07$ (s, 6H). 2c: $78 \%$ yield; ${ }^{1} \mathrm{H}$ NMR $\left(500 \mathrm{MHz}, \mathrm{CDCl}_{3}\right) \delta: 3.66(\mathrm{dd}, J$ $9.8,3.7 \mathrm{~Hz}, 1 \mathrm{H}), 3.40-3.37(\mathrm{~m}$, $1 \mathrm{H}), 2.68-2.65(\mathrm{~m}, 1 \mathrm{H}), 1.55-1.50(\mathrm{~m}, 1 \mathrm{H}), 1.42-1.37(\mathrm{~m}, 1 \mathrm{H}), 1.22-1.13(\mathrm{~m}, 1 \mathrm{H}), 0.92-0.87(\mathrm{~m}, 15 \mathrm{H})$, 0.06 (s, 6H). $2 \mathrm{~d}: 85 \%$ yield; ${ }^{1} \mathrm{H}$ NMR $\left(500 \mathrm{MHz}, \mathrm{CDCl}_{3}\right) \delta: 7.38(\mathrm{dd}, J 8.3,1.3 \mathrm{~Hz}, 2 \mathrm{H}), 7.35-7.32(\mathrm{~m}, 2 \mathrm{H}), 7.28-$ $7.25(\mathrm{~m}, 1 \mathrm{H}), 4.08(\mathrm{dd}, J 8.4,3.9 \mathrm{~Hz}, 1 \mathrm{H}), 3.73(\mathrm{dd}, J 9.8,4.0 \mathrm{~Hz}, 1 \mathrm{H}), 3.53(\mathrm{dd}, J 9.8,8.4 \mathrm{~Hz}, 1 \mathrm{H}), 0.91(\mathrm{~s}, 9 \mathrm{H})$, $0.04(\mathrm{~s}, 3 \mathrm{H}), 0.03(\mathrm{~s}, 3 \mathrm{H})$. The ${ }^{1} \mathrm{H}$ NMR data of $\mathbf{2 d}$ was identical to those reported in the literature. ${ }^{42} \mathbf{2 e :} 88 \%$ yield; ${ }^{1} \mathrm{H}$ NMR $\left(500 \mathrm{MHz}, \mathrm{CDCl}_{3}\right) \delta: 7.32-7.29(\mathrm{~m}, 2 \mathrm{H}), 7.24-7.21(\mathrm{~m}, 3 \mathrm{H}), 3.59(\mathrm{dd}, J 9.7,4.4 \mathrm{~Hz}, 1 \mathrm{H}), 3.45$ (dd, J 9.7, $6.5 \mathrm{~Hz}, 1 \mathrm{H}), 3.13-3.08(\mathrm{~m}, 1 \mathrm{H}), 2.80(\mathrm{dd}, J$ 13.4, $5.3 \mathrm{~Hz}, 1 \mathrm{H}), 2.52(\mathrm{dd}, J 13.4,8.4 \mathrm{~Hz}, 1 \mathrm{H}), 0.92(\mathrm{~s}$, $9 \mathrm{H}), 0.08(\mathrm{~s}, 3 \mathrm{H}), 0.07(\mathrm{~s}, 3 \mathrm{H})$. The ${ }^{1} \mathrm{H}$ NMR data of $2 \mathrm{e}$ was identical to those reported in the literature. ${ }^{43}$

Procedure for the synthesis of compounds $3 a-3 e . \mathrm{Pd}_{2}(\mathrm{dba})_{3}(18 \mathrm{mg}, 0.02 \mathrm{mmol}),( \pm)$-BINAP $(25 \mathrm{mg}, 0.04$ $\mathrm{mmol})$, and sodium tert-butoxide $(250 \mathrm{mg}, 2.6 \mathrm{mmol})$ were dissolved in toluene $(6 \mathrm{ml})$ and the solution degassed for three times before being heated at $95{ }^{\circ} \mathrm{C}$ for $15 \mathrm{~min}$. Upon cooling to room temperature, $2 \mathrm{a}(521$ $\mathrm{mg}, 2.4 \mathrm{mmol}$ ) and 1,2-dibromobenzene (241 $\mathrm{mg}, 2.0 \mathrm{mmol}$ ) were added and the reaction mixture heated at $95{ }^{\circ} \mathrm{C}$ for $2 \mathrm{~h}$. The solution was allowed to cool and filtered through a pad of celite. Solvents were removed under reduced pressure and the crude material purified by column chromatography eluting with light petroleum/ethyl acetate (99/1). Colorless oil (692 mg, 93\%); $\left.{ }^{1} \mathrm{H} \mathrm{NMR} \mathrm{(500} \mathrm{MHz,} \mathrm{CDCl}\right) \delta: 7.41(\mathrm{~d}, J 7.9,1.5 \mathrm{~Hz}$, $1 \mathrm{H}), 7.15-7.12(\mathrm{~m}, 1 \mathrm{H}), 6.65(\mathrm{dd}, J 8.2,1.0 \mathrm{~Hz}, 1 \mathrm{H}), 6.52-6.49(\mathrm{~m}, 1 \mathrm{H}), 4.60(\mathrm{~d}, J 9.1 \mathrm{~Hz}, 1 \mathrm{H}), 3.74(\mathrm{dd}, J 10.1$, $3.7 \mathrm{~Hz}, 1 \mathrm{H}), 3.63(\mathrm{dd}, J 10.1,5.0 \mathrm{~Hz}, 1 \mathrm{H}), 3.28-3.23(\mathrm{~m}, 1 \mathrm{H}), 2.10-2.03(\mathrm{~m}, 1 \mathrm{H}), 1.01(\mathrm{~d}, J 7.0 \mathrm{~Hz}, 3 \mathrm{H}), 1.00(\mathrm{~d}$, J 7.0, 3H), 0.90 (s, 9H), 0.03 (d, J $1.5 \mathrm{~Hz}, 6 \mathrm{H})$.

Analogous compounds $\mathbf{3 b}$-3e were prepared according to the similar procedure for $\mathbf{3 a}$. 3b: $68 \%$ yield; ${ }^{1} \mathrm{H} \mathrm{NMR}$ $\left(500 \mathrm{MHz}, \mathrm{CDCl}_{3}\right) \delta: 7.40(\mathrm{~d}, J 6.5 \mathrm{~Hz}, 1 \mathrm{H}), 7.12(\mathrm{t}, J 7.2 \mathrm{~Hz} 1 \mathrm{H}), 6.70(\mathrm{~d}, J 7.8 \mathrm{~Hz}, 1 \mathrm{H}), 6.49(\mathrm{t}, J 7.0 \mathrm{~Hz}, 1 \mathrm{H}), 4.70$ $(\mathrm{d}, J 9.7 \mathrm{~Hz}, 1 \mathrm{H}), 3.76-3.73(\mathrm{~m}, 2 \mathrm{H}), 3.21-3.19(\mathrm{~m}, 1 \mathrm{H}) 1.26(\mathrm{~s}, 9 \mathrm{H}), 0.86(\mathrm{~s}, 9 \mathrm{H}), 0.01(\mathrm{~s}, 6 \mathrm{H}) .3 \mathrm{c}: 80 \%$ yield; ${ }^{1} \mathrm{H}$ 
NMR $\left(500 \mathrm{MHz}, \mathrm{CDCl}_{3}\right) \delta: 7.41(\mathrm{~d}, J 7.8 \mathrm{~Hz}, 1 \mathrm{H}), 7.13(\mathrm{t}, J 7.7 \mathrm{~Hz}, 1 \mathrm{H}), 6.64(\mathrm{~d}, J 8.2 \mathrm{~Hz}, 1 \mathrm{H}), 6.51(\mathrm{t}, J 7.5 \mathrm{~Hz}, 1 \mathrm{H})$, $4.65(\mathrm{~d}, J 8.9 \mathrm{~Hz}, 1 \mathrm{H}), 3.75$ (dd, J 10.1, 3.6 Hz, 1H), $3.67(\mathrm{dd}, J 10.2,4.6 \mathrm{~Hz}, 1 \mathrm{H}) 3.34-3.31(\mathrm{~m}, 1 \mathrm{H}), 1.82-1.77$ $(\mathrm{m}, 1 \mathrm{H}), 1.65-1.60(\mathrm{~m}, 1 \mathrm{H}), 1.24-1.19(\mathrm{~m}, 1 \mathrm{H}), 0.98-0.93(\mathrm{~m}, 6 \mathrm{H}), 0.90(\mathrm{~s}, 9 \mathrm{H}), 0.03(\mathrm{~s}, 6 \mathrm{H}) .3 \mathrm{~d}: 75 \%$ yield; ${ }^{1} \mathrm{H}$ NMR $\left(500 \mathrm{MHz}, \mathrm{CDCl}_{3}\right) \delta: 7.43(\mathrm{dd}, J 7.9,1.5 \mathrm{~Hz}, 1 \mathrm{H}), 7.38-7.37(\mathrm{~m}, 2 \mathrm{H}), 7.34-7.31(\mathrm{~m}, 2 \mathrm{H}), 7.28-7.26$ $(\mathrm{m}, 1 \mathrm{H}), 6.98-6.94(\mathrm{~m}, 1 \mathrm{H}), 6.54-6.50(\mathrm{~m}, 1 \mathrm{H}), 6.33(\mathrm{dd}, J$ 8.2, $1.4 \mathrm{~Hz}, 1 \mathrm{H}), 5.42(\mathrm{br} \mathrm{s}, 1 \mathrm{H}), 4.44-4.41(\mathrm{~m}$, 1H), 3.95 (dd, J 10.1, 4.2 Hz, 1H), 3.72 (dd, J 10.2, 7.3 Hz, 1H), $0.91(\mathrm{~s}, 9 \mathrm{H}), 0.05$ (s, 3H), 0.01 (s, 3H). 3e: $77 \%$ yield; ${ }^{1} \mathrm{H}$ NMR $\left(500 \mathrm{MHz}, \mathrm{CDCl}_{3}\right) \delta: 7.43(\mathrm{dd}, J 7.9,1.5 \mathrm{~Hz}, 1 \mathrm{H}), 7.33-7.30(\mathrm{~m}, 2 \mathrm{H}), 7.25-7.22(\mathrm{~m}, 3 \mathrm{H}), 7.19-$ $7.16(\mathrm{~m}, 1 \mathrm{H}), 6.70(\mathrm{dd}, J 8.2,1.3 \mathrm{~Hz}, 1 \mathrm{H}), 6.57-6.53(\mathrm{~m}, 1 \mathrm{H}), 4.78(\mathrm{~d}, J 8.9 \mathrm{~Hz}, 1 \mathrm{H}), 3.68-3.58(\mathrm{~m}, 3 \mathrm{H}), 2.94(\mathrm{~d}$, J $6.7 \mathrm{~Hz}, 2 \mathrm{H}), 0.97(\mathrm{~m}, 9 \mathrm{H}), 0.08(\mathrm{~s}, 3 \mathrm{H}), 0.07(\mathrm{~s}, 3 \mathrm{H})$.

Procedure for the synthesis of compounds 4a-4e. Pd(OAc) 2 (34 mg, $0.15 \mathrm{mmol}$ ) and ( \pm )-BINAP (93 mg, 0.15 $\mathrm{mmol}$ ) were dissolved in toluene $(10 \mathrm{~mL})$ under a nitrogen atmosphere, the mixture was heated to $60{ }^{\circ} \mathrm{C}$ for 10 $\mathrm{min}$. After cooling to room temperature, $2 \mathrm{a}$ (556 mg, $1.5 \mathrm{mmol}$ ), 2-naphthylamine ( $258 \mathrm{mg}, 1.8 \mathrm{mmol}$ ), and of $\mathrm{NaO}{ }^{\mathrm{t}} \mathrm{Bu}\left(360 \mathrm{mg} 3.75 \mathrm{mmol}\right.$ ) were added successively under $\mathrm{N}_{2}$. After stirring at $80{ }^{\circ} \mathrm{C}$ for $12 \mathrm{~h}$, the solution was allowed to cool and filtered through a pad of celite. Solvents were removed under reduced pressure and the crude material purified by column chromatography eluting with light petroleum/ethyl acetate (50/1). 4a: yellow oil (566 mg, 87\%); ${ }^{1} \mathrm{H}$ NMR (500 MHz, $\mathrm{CDCl}_{3}$ ) $\delta: 7.70(\mathrm{t}, J 9.3 \mathrm{~Hz}, 2 \mathrm{H}), 7.56$ (d, J $\left.8.3 \mathrm{~Hz}, 1 \mathrm{H}\right), 7.35$ (t, J 7.5 $\mathrm{Hz}, 1 \mathrm{H}), 7.24(\mathrm{t}, J 7.4 \mathrm{~Hz}, 1 \mathrm{H}), 7.18-7.12(\mathrm{~m}, 2 \mathrm{H}), 7.04(\mathrm{~d}, J 8.8 \mathrm{~Hz}, 1 \mathrm{H}), 6.91(\mathrm{~s}, 1 \mathrm{H}), 6.79(\mathrm{~d}, J 7.9 \mathrm{~Hz}, 1 \mathrm{H}), 6.68$ (t, J 7.3 Hz, 1H), 4.43 (br s, 1H), 3.68 (dd, J 10.0, 3.6 Hz, 1H), 3.55 (dd, J 10.0, $5.3 \mathrm{~Hz}, 1 \mathrm{H}), 3.27(\mathrm{~d}, J 4.2 \mathrm{~Hz}, 1 \mathrm{H})$, $2.01-1.97(\mathrm{~m}, 1 \mathrm{H}), 0.92(\mathrm{~d}, J 6.7 \mathrm{~Hz}, 3 \mathrm{H}), 0.87(\mathrm{~d}, J 6.7 \mathrm{~Hz}, 3 \mathrm{H}), 0.80(\mathrm{~s}, 9 \mathrm{H}),-0.03(\mathrm{~s}, 3 \mathrm{H}),-0.08(\mathrm{~s}, 3 \mathrm{H})$.

Analogous compounds $\mathbf{4 b - 4 e}$ were prepared according to the similar procedure for $\mathbf{4 a}$. $4 \mathbf{b}$ : $91 \%$ yield; ${ }^{1} \mathrm{H}$ NMR $\left(500 \mathrm{MHz}, \mathrm{CDCl}_{3}\right) \delta: 7.70-7.67(\mathrm{~m}, 2 \mathrm{H}), 7.55(\mathrm{~d}, J 8.3 \mathrm{~Hz}, 1 \mathrm{H}), 7.34(\mathrm{t}, J 7.1 \mathrm{~Hz}, 1 \mathrm{H}), 7.22(\mathrm{t}, J 7.0 \mathrm{~Hz}, 1 \mathrm{H}), 7.16-$ $7.10(\mathrm{~m}, 2 \mathrm{H}), 7.04(\mathrm{dd}, J$ 8.7, $2.1 \mathrm{~Hz}, 1 \mathrm{H}), 6.91(\mathrm{~s}, 1 \mathrm{H}), 6.82(\mathrm{~d}, J 8.1 \mathrm{~Hz}, 1 \mathrm{H}), 6.67-6.64(\mathrm{~m}, 1 \mathrm{H}), 3.71-3.64(\mathrm{~m}$, 2H), $3.18-3.16(\mathrm{~m}, 1 \mathrm{H}), 0.91(\mathrm{~s}, 9 \mathrm{H}), 0.79(\mathrm{~s}, 9 \mathrm{H}),-0.04(\mathrm{~s}, 3 \mathrm{H}),-0.09(\mathrm{~s}, 3 \mathrm{H}) .4 \mathrm{c}: 98 \%$ yield; ${ }^{1} \mathrm{H} \mathrm{NMR}(500 \mathrm{MHz}$, $\left.\mathrm{CDCl}_{3}\right) \delta: 7.70(\mathrm{t}, J 8.5 \mathrm{~Hz}, 2 \mathrm{H}), 7.57(\mathrm{~d}, J 8.1 \mathrm{~Hz}, 1 \mathrm{H}), 7.36(\mathrm{t}, J 7.4 \mathrm{~Hz}, 1 \mathrm{H}), 7.28-7.23(\mathrm{~m}, 3 \mathrm{H}), 7.09-7.06(\mathrm{~m}$, $2 \mathrm{H}), 6.99(\mathrm{~s}, 1 \mathrm{H}), 6.84-8.82(\mathrm{~m}, 1 \mathrm{H}), 3.72-3.68(\mathrm{~m}, 2 \mathrm{H}), 3.44-3.37(\mathrm{~m}, 1 \mathrm{H}), 1.85-1.76(\mathrm{~m}, 1 \mathrm{H}), 1.65-1.56$ $(\mathrm{m}, 1 \mathrm{H}), 1.53-1.46(\mathrm{~m}, 1 \mathrm{H}), 0.96-0.86(\mathrm{~m}, 6 \mathrm{H}), 0.81(\mathrm{~s}, 9 \mathrm{H}),-0.02(\mathrm{~s}, 3 \mathrm{H}),-0.06(\mathrm{~s}, 3 \mathrm{H}) .4 \mathrm{~d}: 99 \%$ yield; ${ }^{1} \mathrm{H}$ NMR $\left(500 \mathrm{MHz} \mathrm{CDCl}_{3}\right) \delta: 7.73(\mathrm{dd}, J 8.2,5.2 \mathrm{~Hz}, 2 \mathrm{H}), 7.61(\mathrm{~d}, J 8.2 \mathrm{~Hz}, 1 \mathrm{H}), 7.40-7.38(\mathrm{~m}, 3 \mathrm{H}), 7.32(\mathrm{t}, J 7.4 \mathrm{~Hz}$, $2 \mathrm{H}), 7.27-7.24(\mathrm{~m}, 2 \mathrm{H}), 7.19(\mathrm{~d}, J 7.3 \mathrm{~Hz}, 1 \mathrm{H}), 7.11(\mathrm{dd}, J 8.7,2.0 \mathrm{~Hz}, 1 \mathrm{H}), 6.98(\mathrm{~s}, 1 \mathrm{H}), 6.95(\mathrm{t}, J 7.9 \mathrm{~Hz}, 1 \mathrm{H})$, $6.72(\mathrm{t}, J 7.4 \mathrm{~Hz}, 1 \mathrm{H}), 6.52(\mathrm{~d}, J 7.3 \mathrm{~Hz}, 1 \mathrm{H}), 4.44-4.41(\mathrm{~m}, 1 \mathrm{H}), 3.85(\mathrm{dd}, J 10.1,4.1 \mathrm{~Hz}, 1 \mathrm{H}), 3.65-3.61(\mathrm{~m}, 1 \mathrm{H})$, $0.70(\mathrm{~s}, 9 \mathrm{H}),-0.13(\mathrm{~s}, 3 \mathrm{H}),-0.19(\mathrm{~s}, 3 \mathrm{H}) .4 \mathrm{e}: 97 \%$ yield; ${ }^{1} \mathrm{H}$ NMR $\left(500 \mathrm{MHz}, \mathrm{CDCl}_{3}\right) \delta: 7.72-7.68(\mathrm{~m}, 2 \mathrm{H}), 7.55$ $(\mathrm{d}, J 8.2 \mathrm{~Hz}, 1 \mathrm{H}), 7.36(\mathrm{t}, J 7.1 \mathrm{~Hz}, 1 \mathrm{H}), 7.26-7.14(\mathrm{~m}, 8 \mathrm{H}), 6.99(\mathrm{dd}, J 8.7,1.8 \mathrm{~Hz}, 1 \mathrm{H}), 6.87-6.86(\mathrm{~m}, 2 \mathrm{H}), 6.74$ (t, J $7.1 \mathrm{~Hz}, 1 \mathrm{H}$ ), 3.67 (br s, 1H), 3.59 (dd, J 9.9, $3.1 \mathrm{~Hz}, 1 \mathrm{H}), 3.50$ (dd, J 9.9, $4.8 \mathrm{~Hz}, 1 \mathrm{H}), 2.90-2.84(\mathrm{~m}, 2 \mathrm{H}), 0.80$ $(\mathrm{s}, 9 \mathrm{H}),-0.04(\mathrm{~s}, 3 \mathrm{H}),-0.10(\mathrm{~s}, 3 \mathrm{H})$.

Procedure for the synthesis of compounds 5a-5e. Diamine 4 a $(781 \mathrm{mg}, 1.8 \mathrm{mmol}$ ) was dissolved in $70 \mathrm{~mL}$ triethylorthoformate, then concentrated hydrochloric acid ( $37 \% \mathrm{w} / \mathrm{w}, 10.8 \mathrm{mmol}, 896 \mu \mathrm{L}$ of solution) was added at room temperature and the mixture was stirred for 30 mins. Then the mixture was heated to $80{ }^{\circ} \mathrm{C}$ under air atmosphere for $12 \mathrm{~h}$. After cooling to room temperature, solvents were removed under reduced pressure and the crude material purified by column chromatography eluting with $\mathrm{CH}_{2} \mathrm{Cl}_{2} / \mathrm{MeOH}=45: 1$, giving the product $5 \mathrm{a}$ as white solid (560 mg, 85\%). ${ }^{1} \mathrm{H}$ NMR $(500 \mathrm{MHz}, \mathrm{DMSO}) \delta: 10.47(\mathrm{~s}, 1 \mathrm{H}), 8.55(\mathrm{~s}, 1 \mathrm{H}), 8.33$ (d, J $8.7 \mathrm{~Hz}, 2 \mathrm{H}), 8.16(\mathrm{~d}, J 2.7 \mathrm{~Hz}, 2 \mathrm{H}), 8.02-7.96(\mathrm{~m}, 2 \mathrm{H}), 7.81-7.73(\mathrm{~m}, 4 \mathrm{H}), 5.46(\mathrm{t}, J 5.8 \mathrm{~Hz}, 1 \mathrm{H}), 4.82-4.81(\mathrm{~m}$, $1 \mathrm{H}), 4.12-4.07(\mathrm{~m}, 1 \mathrm{H}), 3.92-3.90(\mathrm{~m}, 1 \mathrm{H}), 2.56-2.50(\mathrm{~m}, 1 \mathrm{H}), 1.15(\mathrm{~d}, J 6.5 \mathrm{~Hz}, 3 \mathrm{H}), 0.88(\mathrm{t}, J 7.8 \mathrm{~Hz}, 3 \mathrm{H})$; 
${ }^{13} \mathrm{C}$ NMR (125 MHz, DMSO) $\delta: 142.3,133.2,130.7,128.9,128.5,128.4,128.2,128.0,127.5,125.1,123.3$, 114.9, 114.2, 67.5, 60.7, 19.8, 19.7; HR-ESIMS: $\mathrm{m} / \mathrm{z} 331.1823[\mathrm{M}-\mathrm{Cl}]^{+}$(calcd for $\mathrm{C}_{22} \mathrm{H}_{23} \mathrm{~N}_{2} \mathrm{O}, 331.1805$ ).

Analogous compounds $\mathbf{5 b}-\mathbf{5 e}$ were prepared according to the similar procedure for $\mathbf{5 a}$. $\mathbf{5 b}$ : $83 \%$ yield; ${ }^{1} \mathrm{H}$ NMR (500 MHz, DMSO) $\delta: 10.52(\mathrm{~s}, 1 \mathrm{H}), 8.57(\mathrm{~s}, 1 \mathrm{H}), 8.42(\mathrm{~d}, J 8.4 \mathrm{~Hz}, 1 \mathrm{H}), 8.32(\mathrm{~d}, J 8.8 \mathrm{~Hz}, 1 \mathrm{H}), 8.17-8.15(\mathrm{~m}, 2 \mathrm{H})$, $8.02(\mathrm{dd}, J$ 8.7, $1.9 \mathrm{~Hz}, 1 \mathrm{H}), 7.95(\mathrm{~d}, J 8.2 \mathrm{~Hz}, 1 \mathrm{H}), 7.81-7.76(\mathrm{~m}, 1 \mathrm{H}), 7.75-7.72(\mathrm{~m}, 3 \mathrm{H}), 5.38(\mathrm{t}, J 5.6 \mathrm{~Hz}, 1 \mathrm{H})$, $4.96-4.94(\mathrm{~m}, 1 \mathrm{H}), 4.26(\mathrm{br} \mathrm{s}, 1 \mathrm{H}), 4.11-4.07(\mathrm{~m}, 1 \mathrm{H}), 1.07(\mathrm{~s}, 9 \mathrm{H}) ;{ }^{13} \mathrm{C} \mathrm{NMR}(125 \mathrm{MHz}, \mathrm{DMSO}) \delta: 142.2$, $134.3,133.6,133.2,131.3,131.1,130.6,128.9,128.5,128.5,128.2,127.9,127.5,125.3,123.5,115.2,114.1$, 70.1, 59.5, 55.4, 35.0; HR-ESIMS: $m / z$ 345.1972 [M-Cl] ${ }^{+}$(calcd for $\mathrm{C}_{23} \mathrm{H}_{25} \mathrm{~N}_{2} \mathrm{O}, 345.1961$ ). 5c: 87\% yield; ${ }^{1} \mathrm{H} N M R$ (500 MHz, DMSO) $\delta: 10.44(\mathrm{~s}, 1 \mathrm{H}), 8.52(\mathrm{~s}, 1 \mathrm{H}), 8.33-8.30(\mathrm{~m}, 2 \mathrm{H}), 8.16-8.15(\mathrm{~m}, 2 \mathrm{H}), 8.00-7.96(\mathrm{~m}, 2 \mathrm{H})$, $7.81-7.72(\mathrm{~m}, 4 \mathrm{H}), 5.40(\mathrm{t}, J 5.9 \mathrm{~Hz}, 1 \mathrm{H}), 4.88-4.85(\mathrm{~m}, 1 \mathrm{H}), 4.11-4.06(\mathrm{~m}, 1 \mathrm{H}), 3.91-3.87(\mathrm{~m}, 1 \mathrm{H}), 2.37-$ $2.35(\mathrm{~m}, 1 \mathrm{H}), 1.36-1.32(\mathrm{~m}, 1 \mathrm{H}), 1.16-1.13(\mathrm{~m}, 1 \mathrm{H}), 1.11(\mathrm{~d}, J 6.7 \mathrm{~Hz}, 3 \mathrm{H}), 0.83(\mathrm{t}, J 7.3 \mathrm{~Hz}, 3 \mathrm{H}) ;{ }^{13} \mathrm{C} N \mathrm{NMR}(125$ MHz, DMSO) $\delta: 142.3,133.6,133.2,132.7,131.7,131.2,130.7,128.9,128.5,128.4,128.2,128.0,127.5,125.1$, 123.3, 114.9, 114.3, 65.9, 60.6, 35.1, 25.4, 15.6, 11.0; HR-ESIMS: $m / z$ 345.1974 [M-Cl] ${ }^{+}$(calcd for $\mathrm{C}_{23} \mathrm{H}_{25} \mathrm{~N}_{2} \mathrm{O}$, 345.1961). 5d: 93\% yield; ${ }^{1} \mathrm{H}$ NMR (500 MHz, DMSO) $\delta: 10.69$ (s, 1H), 8.59 (d, J $\left.2.0 \mathrm{~Hz}, 1 \mathrm{H}\right), 8.33(\mathrm{~d}, J 8.9 \mathrm{~Hz}$, $1 \mathrm{H}), 8.18-8.15(\mathrm{~m}, 2 \mathrm{H}), 8.08-8.04(\mathrm{~m}, 2 \mathrm{H}), 7.98-7.96(\mathrm{~m}, 1 \mathrm{H}), 7.75-7.71(\mathrm{~m}, 4 \mathrm{H}), 7.69-7.68(\mathrm{~m}, 2 \mathrm{H}), 7.46$ $-7.40(\mathrm{~m}, 3 \mathrm{H}), 6.29-6.27(\mathrm{~m}, 1 \mathrm{H}), 5.90(\mathrm{t}, J 5.7 \mathrm{~Hz}, 1 \mathrm{H}) 4.60-4.54(\mathrm{~m}, 1 \mathrm{H}), 4.23-4.18(\mathrm{~m}, 1 \mathrm{H}) ;{ }^{13} \mathrm{C} \mathrm{NMR}(125$ $\mathrm{MHz}$, DMSO) $\delta: 142.4,135.5,133.6,133.2,131.9,131.8,131.1,130.7,129.4,128.9,128.5,128.5,128.4,128.2$, $128.2,128.1,127.7,125.2,123.2,114.9,114.4,112.5,64.6,62.5$, one resonance was not observed due to coincidental overlap; HR-ESIMS: $\mathrm{m} / z 365.1652$ [M-Cl] ${ }^{+}$(calcd for $\mathrm{C}_{25} \mathrm{H}_{21} \mathrm{~N}_{2} \mathrm{O}, 365.1648$ ) 5e: 93\% yield; ${ }^{1} \mathrm{H}$ NMR (500 MHz, DMSO) $\delta: 10.63(\mathrm{~s}, 1 \mathrm{H}), 8.48(\mathrm{~d}, J 2.0 \mathrm{~Hz}, 1 \mathrm{H}), 8.32(\mathrm{~d}, J 8.8 \mathrm{~Hz}, 1 \mathrm{H}), 8.22(\mathrm{~d}, J 7.8 \mathrm{~Hz}, 1 \mathrm{H}), 8.17-8.15$ $(\mathrm{m}, 2 \mathrm{H}), 7.93(\mathrm{dd}, J 8.7,2.2 \mathrm{~Hz}, 1 \mathrm{H}), 7.90(\mathrm{~d}, J 7.6 \mathrm{~Hz}, 1 \mathrm{H}), 7.75-7.67(\mathrm{~m}, 4 \mathrm{H}), 7.36(\mathrm{~d}, J 7.3 \mathrm{~Hz}, 2 \mathrm{H}), 7.27-7.24$ $(\mathrm{m}, 2 \mathrm{H}), 7.19-7.16(\mathrm{~m}, 1 \mathrm{H}), 5.62(\mathrm{t}, J 6.2 \mathrm{~Hz}, 1 \mathrm{H}), 5.39-5.37(\mathrm{~m}, 1 \mathrm{H}), 4.01-3.96(\mathrm{~m}, 1 \mathrm{H}), 3.92-3.87(\mathrm{~m}, 1 \mathrm{H})$, $3.54-3.50(\mathrm{~m}, 1 \mathrm{H}), 3.46-3.42(\mathrm{~m}, 1 \mathrm{H}) ;{ }^{13} \mathrm{C} N M R(125 \mathrm{MHz}, \mathrm{DMSO}) \delta: 142.5,137.2,133.6,133.2,132.3,131.3$, 131.0, 130.8, 129.6, 129.0, 128.9, 128.5, 128.5, 128.3, 127.9, 127.4, 127.3, 124.9, 123.0, 114.8, 114.0, 79.8, 62.6, 61.9, 36.1; HR-ESIMS: $m / z 379.1812[\mathrm{M}-\mathrm{Cl}]^{+}$(calcd for $\mathrm{C}_{26} \mathrm{H}_{23} \mathrm{~N}_{2} \mathrm{O}, 379.1805$ ).

Procedure for the synthesis of compounds 6a-6e. To a solution of imidazolinium salt 5a (366.0 mg, 1.00 $\mathrm{mmol}$ ) in $\mathrm{CH}_{2} \mathrm{Cl}_{2}(25 \mathrm{~mL}$ ) was added silver(I) oxide (115.9 mg, $0.50 \mathrm{mmol})$. The suspension was stirred for $3 \mathrm{~h}$ in the darkness, during which the black color diminished gradually. The reaction mixture was filtered through a small pad of Celite, $[\mathrm{Rh}(\mathrm{COD}) \mathrm{Cl}]_{2}(246.5 \mathrm{mg}, 0.50 \mathrm{mmol})$ was added, and the reaction mixture was stirred for an additional $16 \mathrm{~h}$. The solvents were removed under reduced pressure, and the residue was purified by flash chromatography on silica gel with $\mathrm{CH}_{2} \mathrm{Cl}_{2}$ as eluent to give $6 \mathrm{a}\left(379.4 \mathrm{mg}, 70 \%\right.$ yield). ${ }^{1} \mathrm{H} \mathrm{NMR}\left(500 \mathrm{MHz} \mathrm{CDCl}_{3}\right)$ $\delta: ~ 8.73(\mathrm{br} \mathrm{s}, 1 \mathrm{H}), 8.13-8.01(\mathrm{~m}, 3 \mathrm{H}), 7.66-7.62(\mathrm{~m}, 2 \mathrm{H}), 7.59-7.57(\mathrm{~m}, 1 \mathrm{H}), 7.44-7.42(\mathrm{~m}, 1 \mathrm{H}), 7.31-7.20$ $(\mathrm{m}, 3 \mathrm{H}), 5.90-5.85(\mathrm{~m}, 1 \mathrm{H}), 5.10-4.97(\mathrm{~m}, 2 \mathrm{H}), 4.37-4.35(\mathrm{~m}, 1 \mathrm{H}), 4.23(\mathrm{dd}, J 10.1,3.1 \mathrm{~Hz}, 1 \mathrm{H}), 4.14-4.08$ $(\mathrm{m}, 1 \mathrm{H}), 3.55-3.52(\mathrm{~m}, 1 \mathrm{H}), 2.73-2.67(\mathrm{~m}, 2 \mathrm{H}), 2.38-2.24(\mathrm{~m}, 2 \mathrm{H}), 1.87-1.85(\mathrm{~m}, 1 \mathrm{H}), 1.79-1.74(\mathrm{~m}, 1 \mathrm{H})$, $1.60-1.57(\mathrm{~m}, 1 \mathrm{H}), 1.36(\mathrm{~d}, J 6.5 \mathrm{~Hz}, 3 \mathrm{H}), 1.35-1.26(\mathrm{~m}, 1 \mathrm{H}), 1.05(\mathrm{~d}, J 6.5 \mathrm{~Hz}, 3 \mathrm{H}), 1.04-0.98(\mathrm{~m}, 1 \mathrm{H})$; HRESIMS: $m / z 541.1733[\mathrm{M}-\mathrm{Cl}]^{+}$(calcd for $\mathrm{C}_{30} \mathrm{H}_{34} \mathrm{~N}_{2} \mathrm{ORh}, 541.1726$ ).

Analogous compounds $6 \mathbf{b}-6 \mathrm{e}$ were prepared according to the similar procedure for $6 \mathrm{a} .6 \mathrm{~b}: 73 \%$ yield; ${ }^{1} \mathrm{H}$ NMR $\left(500 \mathrm{MHz}, \mathrm{CDCl}_{3}\right) \delta: 8.82(\mathrm{br} \mathrm{s}, 1 \mathrm{H}), 8.12-8.01(\mathrm{~m}, 3 \mathrm{H}), 7.66-7.62(\mathrm{~m}, 3 \mathrm{H}), 7.34-7.16(\mathrm{~m}, 3 \mathrm{H}), 6.21-6.18(\mathrm{~m}$, $1 \mathrm{H}), 5.06-4.97(\mathrm{~m}, 2 \mathrm{H}), 4.38-4.20(\mathrm{~m}, 3 \mathrm{H}), 3.61-3.58(\mathrm{~m}, 1 \mathrm{H}), 2.88-2.80(\mathrm{~m}, 1 \mathrm{H}), 2.39-2.21(\mathrm{~m}, 2 \mathrm{H}), 1.88$ $-1.70(\mathrm{~m}, 2 \mathrm{H}), 1.45-1.40(\mathrm{~m}, 1 \mathrm{H}), 1.35(\mathrm{~s}, 9 \mathrm{H}), 1.31-1.25(\mathrm{~m}, 2 \mathrm{H}), 0.95-0.87(\mathrm{~m}, 1 \mathrm{H}) ; \mathrm{HR}-\mathrm{ESIMS}: m / z$ $555.1895[\mathrm{M}-\mathrm{Cl}]^{+}$(calcd for $\left.\mathrm{C}_{31} \mathrm{H}_{36} \mathrm{~N}_{2} \mathrm{ORh}, 555.1883\right) .6 \mathrm{c}: 71 \%$ yield; ${ }^{1} \mathrm{H}$ NMR $\left(500 \mathrm{MHz}, \mathrm{CDCl}_{3}\right) \delta: 8.64(\mathrm{br} \mathrm{s}, 1 \mathrm{H})$, $8.04-7.93(\mathrm{~m}, 3 \mathrm{H}), 7.58-7.54(\mathrm{~m}, 2 \mathrm{H}), 7.50-7.48(\mathrm{~m}, 1 \mathrm{H}), 7.36-7.34(\mathrm{~m}, 1 \mathrm{H}), 7.22-7.12(\mathrm{~m}, 3 \mathrm{H}), 5.88-$ $5.83(\mathrm{~m}, 1 \mathrm{H}), 5.01-4.88(\mathrm{~m}, 2 \mathrm{H}), 4.30-4.26(\mathrm{~m}, 1 \mathrm{H}), 4.13-4.10(\mathrm{~m}, 1 \mathrm{H}), 4.07-4.00(\mathrm{~m}, 1 \mathrm{H}), 3.45-3.42(\mathrm{~m}$, 
$1 \mathrm{H}), 2.65-2.63(\mathrm{~m}, 1 \mathrm{H}), 2.30-2.22(\mathrm{~m}, 4 \mathrm{H}), 1.95-1.92(\mathrm{~m}, 1 \mathrm{H}), 1.77-1.75(\mathrm{~m}, 1 \mathrm{H}), 1.68-1.65(\mathrm{~m}, 1 \mathrm{H}), 1.52$ $-1.49(\mathrm{~m}, 1 \mathrm{H}), 1.34-1.31(\mathrm{~m}, 1 \mathrm{H}), 1.24(\mathrm{~d}, J 6.5 \mathrm{~Hz}, 3 \mathrm{H}), 1.18(\mathrm{~s}, 9 \mathrm{H}), 0.87(\mathrm{t}, J 7.3 \mathrm{~Hz}, 3 \mathrm{H}), 0.76-0.73(\mathrm{~m}, 1 \mathrm{H})$; HR-ESIMS: $m / z 555.1892[\mathrm{M}-\mathrm{Cl}]^{+}$(calcd for $\left.\mathrm{C}_{31} \mathrm{H}_{36} \mathrm{~N}_{2} \mathrm{ORh}, 555.1883\right)$ ) $6 \mathrm{~d}$ : $74 \%$ yield; ${ }^{1} \mathrm{H} \mathrm{NMR}\left(500 \mathrm{MHz} \mathrm{CDCl}_{3}\right)$ $\delta: 8.78(\mathrm{br} \mathrm{s}, 1 \mathrm{H}), 8.16-8.14(\mathrm{~m}, 1 \mathrm{H}), 8.09-8.03(\mathrm{~m}, 2 \mathrm{H}), 7.85-7.82(\mathrm{~m}, 1 \mathrm{H}), 7.67-7.64(\mathrm{~m}, 2 \mathrm{H}), 7.51-7.49$ $(\mathrm{m}, 1 \mathrm{H}), 7.44-7.38(\mathrm{~m}, 5 \mathrm{H}), 7.25-7.15(\mathrm{~m}, 3 \mathrm{H}), 5.17-5.13(\mathrm{~m}, 1 \mathrm{H}), 4.98-4.94(\mathrm{~m}, 1 \mathrm{H}), 4.78-4.74(\mathrm{~m}, 1 \mathrm{H})$, $4.48(\mathrm{dd}, J 22.5,11.0 \mathrm{~Hz}, 1 \mathrm{H}), 4.21(\mathrm{dd}, J 11.0,2.0 \mathrm{~Hz}, 1 \mathrm{H}), 3.37-3.34(\mathrm{~m}, 1 \mathrm{H}), 2.70-2.68(\mathrm{~m}, 1 \mathrm{H}), 2.15-2.00$ $(\mathrm{m}, 2 \mathrm{H}), 1.72-1.59(\mathrm{~m}, 4 \mathrm{H}), 1.32-1.25(\mathrm{~m}, 1 \mathrm{H}), 1.07-0.99(\mathrm{~m}, 1 \mathrm{H}) ; \mathrm{HR}-\mathrm{ESIMS}: \mathrm{m} / z 575.1586$ [M-Cl] $^{+}$(calcd for $\left.\mathrm{C}_{33} \mathrm{H}_{32} \mathrm{~N}_{2} \mathrm{ORh}, 575.1570\right)$. 6e: $69 \%$ yield; ${ }^{1} \mathrm{H}$ NMR $\left(500 \mathrm{MHz}, \mathrm{CDCl}_{3}\right) \delta: 8.70(\mathrm{br} \mathrm{s}, 1 \mathrm{H}), 8.11-8.09(\mathrm{~m}, 1 \mathrm{H})$, $8.05-8.00(\mathrm{~m}, 2 \mathrm{H}), 7.75-7.74(\mathrm{~m}, 1 \mathrm{H}), 7.64-7.60(\mathrm{~m}, 2 \mathrm{H}), 7.45-7.44(\mathrm{~m}, 1 \mathrm{H}), 7.39-7.19(\mathrm{~m}, 7 \mathrm{H}), 6.51-$ $6.45(\mathrm{~m}, 1 \mathrm{H}), 5.06-5.02(\mathrm{~m}, 1 \mathrm{H}), 4.91-4.87(\mathrm{~m}, 1 \mathrm{H}), 4.33-4.22(\mathrm{~m}, 2 \mathrm{H}), 4.11(\mathrm{dd}, J 9.9,2.7 \mathrm{~Hz}, 1 \mathrm{H}), 3.57(\mathrm{dd}$, J 13.9, $4.8 \mathrm{~Hz}, 1 \mathrm{H}), 3.43(\mathrm{dd}, J 14.0,7.2 \mathrm{~Hz}, 1 \mathrm{H}), 2.60-2.53(\mathrm{~m}, 2 \mathrm{H}), 2.27-2.19(\mathrm{~m}, 1 \mathrm{H}), 2.11-2.04(\mathrm{~m}, 1 \mathrm{H})$, $1.73-1.69(\mathrm{~m}, 1 \mathrm{H}), 1.63-1.56(\mathrm{~m}, 1 \mathrm{H}), 1.48-1.47(\mathrm{~m}, 2 \mathrm{H}), 1.26-1.23(\mathrm{~m}, 1 \mathrm{H}), 0.88-0.82(\mathrm{~m}, 1 \mathrm{H})$; HRESIMS: $m / z 589.1739[\mathrm{M}-\mathrm{Cl}]^{+}$(calcd for $\left.\mathrm{C}_{34} \mathrm{H}_{34} \mathrm{~N}_{2} \mathrm{ORh}, 589.1726\right)$.

Anti-tumor activity. The MCF-7 cells were purchased from American Type Culture Collection (ATCC, Manassas, VA, USA). They were routinely maintained in the RPMI-1640 with $10 \% \mathrm{FBS}$ at $37{ }^{\circ} \mathrm{C}$ and an atmosphere of $5 \%$ $\mathrm{CO}_{2}$. The antitumor activities of the compounds were determined by MTT assay. Cells were seeded in 96-well cell culture plates at a density of 10000 cells/well in $100 \mu \mathrm{L}$ of culture medium, incubated at $37{ }^{\circ} \mathrm{C}$ and $5 \% \mathrm{CO}_{2}$. After $24 \mathrm{~h}$ incubation, they were treated with different concentrations of the candidate compounds for the indicated time periods, respectively. Cell viability was measured by the 3-(4, 5-dimetrylthiazol-2-yl)-2, 5diphenyltetrazolium bromide (MTT) assay.

\section{Acknowledgements}

We are grateful to the National Natural Science Foundation of China (81302668) and Hangzhou Science and Technology Information Institute of China (20150633B45).

\section{Supplementary Material}

NMR spectra of $5 a-5 e$ and $6 a-6 e$.

\section{References}

1. Öfele, K. J. Organomet. Chem. 1968, 12, 42-45. https://doi.org/10.1016/S0022-328X(00)88691-X

2. Wanzlick, H. W.; Schönherr, H. J. Angew. Chem., Int. Ed. Engl. 1968, 7, 141-142. https://doi.org/10.1002/anie.196801412

3. Arduengo, A. J.; Harlow, R. L.; Kline, M. J. Am. Chem. Soc. 1991, 113, 361-363. https://doi.org/10.1021/ja00001a054

4. Herrmann, W. A.; Köcher, C. Angew. Chem., Int. Ed. 1997, 36, 2162-2187. https://doi.org/10.1002/anie.199721621 
5. Bourissou, D.; Guerret, O.; Gabbaï, F. P.; Bertrand, G. Chem. Rev. 2000, 100, 39-92. https://doi.org/10.1021/cr940472u

6. Zinn, F. K.; Viciu, M. S.; Nolan, S. P. Annu. Rep. Prog. Chem. Sect. B 2004, 100, 231-249. https://doi.org/10.1039/B401751J

7. Cavell, K. J.; McGuinness, D. S. Coord. Chem. Rev. 2004, 248, 671-681. https://doi.org/10.1016/j.ccr.2004.02.006

8. Peris, E.; Crabtree, R. H. Coord. Chem. Rev. 2004, 248, 2239-2246. https://doi.org/10.1016/i.ccr.2004.04.014

9. Crudden, C. M.; Allen, D. P. Coord. Chem. Rev. 2004, 248, 2247-2273. https://doi.org/10.1016/i.ccr.2004.05.013

10. Pugh, D.; Danopoulos, A. A. Coord. Chem. Rev. 2007, 251, 610-641. https://doi.org/10.1016/i.ccr.2006.08.001

11. Mata, J. A.; Poyatos, M.; Peris, E. Coord. Chem. Rev. 2007, 251, 841-859. https://doi.org/10.1016/i.ccr.2006.06.008

12. Sommer, W. J.; Weck, M. Coord. Chem. Rev. 2007, 251, 860-873. https://doi.org/10.1016/j.ccr.2006.07.004

13. Hahn, F. E.; Jahnke, M. C. Angew. Chem., Int. Ed. 2008, 47, 3122-3172. https://doi.org/10.1002/anie.200703883

14. Hopkinson, M. N.; Richter, C.; Schedler, M.; Glorius, F. Nature 2014, 510, 485-496. https://doi.org/10.1038/nature13384

15. Zou, T.; Hung, F. F.; Yang, C.; Che, C. M. Strongly phosphorescent transition-metal complexes with Nheterocyclic carbene ligands as cellular probes. In Luminescent and Photoactive Transition Metal Complexes as Biomolecular Probes and Cellular Reagents. Structure and Bonding, vol 165. Edited by KamWing Lo K . Berlin Heidelberg: Springer; 2015:181-203.

https://doi.org/10.1007/430 2015173

16. Barnard, P. J.; Baker, M. V.; Berners-Price, S. J.; Day, D.A. J. Inorg. Biochem. 2004, 98,1642-1647. https://doi.org/10.1016/j.jinorgbio.2004.05.011

17. Karaca, O.; Meier-Menches, S. M.; Casini, A.; Kuhn, F. E. Chem. Commun. 2017, 53, 8249-8260. https://doi.org/10.1039/C7CC03074F

18. Liu, W. K.; Gust, R. Coord. Chem. Rev. 2016, 329, 191-213.

https://doi.org/10.1016/i.ccr.2016.09.004

19. Hemmert, C.; Gornitzka, H. Dalton Trans. 2016, 45, 440-447. https://doi.org/10.1039/C5DT03904E

20. Cisnetti, F.; Gautier, A. Angew. Chem., Int. Ed. 2013, 52, 11976-11978.

https://doi.org/10.1002/anie.201306682

21. Oehninger, L.; Rubbiani, R.; Ott, I. Dalton Trans. 2013, 42, 3269-3284. https://doi.org/10.1039/C2DT32617E

22. Teyssot, M. L.; Jarrousse, A. S.; Manin, M.; Chevry, A.; Roche, S.; Norre, F.; Beaudoin, C.; Morel, L.; Boyer, D.; Mahiou, R. Dalton Trans. 2009, 35, 6894-6902.

https://doi.org/10.1039/b906308k

23. Li, J.; Xu, L. Tetrahedron 2015, 71, 2858-2862. https://doi.org/10.1016/i.tet.2015.03.067

24. Zhou, B. H.; Wu, C.; Chen, X. X.; Huang, H. X.; Li, L. L.; Fan, L. M.; Li, J. Tetrahedron Lett. 2017, 58, 41574161. 
https://doi.org/10.1016/i.tetlet.2017.09.022

25. Zhou, B. H.; Li, Z. J.; Yang, C.; Li, L. L.; Fan, L. M.; Huang, H. X.; Li, J. Arkivoc 2018, (v), 1-10. https://doi.org/10.1016/i.tetlet.2017.09.022

26. Zhou, B. H.; He, W. P.; Li, L. L.; Fan, L. M.; Li, X. R.; Li, J. Curr. Org. Syn. 2018, 15, 137-142. https://doi.org/10.2174/1570179414666170830130658

27. Li, J.; Yao, J. Q.; He, W. P.; Yang, F.; Liu, X. M. Lett. Org. Chem. 2019, 16, 951-954. https://doi.org/10.2174/1570178616666190212125426

28. Li, J.; Zhou, B. H.; Jiang, Y. J.; Liu, X. M. Catalysts 2018, 8, 46. https://doi.org/10.3390/catal8020046

29. He, W. P.; Zhou, B. H.; Zhou, Y. L.; Li, X. R.; Fan, L. M.; Shou, H. W.; Li, J. Tetrahedron Lett. 2016, 57, 31523155.

https://doi.org/10.1016/i.tetlet.2016.06.023

30. He, W. P.; Zhao, W.; Zhou, B. H.; Liu, H. F.; Li, X. R.; Li, L. L.; Li, J.; Shi, J. Y. Molecules 2016, $21,742$. https://doi.org/10.3390/molecules21060742

31. He, W. P.; Zhou, B. H.; Li, J.; Shi, J. Y. Catalysts 2016, 6, 132. https://doi.org/10.3390/catal6090132

32. Dorcier, A.; Ang, W. H.; Bolaño, S.; Gonsalvi, L.; Juillerat-Jeannerat, L.; Laurenczy, G.; Peruzzini, M.; Phillips, A. D.; Zanobini, F.; Dyson, P. J. Organometallics 2006, 25, 4090-4096. https://doi.org/10.1021/om0603940

33. Angeles-Boza, A. M.; Chifotides, H. T.; Aguirre, J. D.; Chouai, A.; Fu, P. K. L.; Dunbar, K. R.; Turro, C. J. Med. Chem. 2006, 49, 6841-6847. https://doi.org/10.1021/jm060592h

34. Aguirre, J. D.; Angeles-Boza, A. M.; Chouai, A.; Turro, C.; Pellois, J.-P.; Dunbar, K. R. Dalton Trans. 2009, 10806-10812. https://doi.org/10.1039/b915357h

35. Bieda, R.; Dobroschke, M.; Triller, A.; Ott, I.; Spehr, M.; Gust, R.; Prokop, A.; Sheldrick, W. S. ChemMedChem 2010, 5, 1123-1133.

https://doi.org/10.1002/cmdc.201000129

36. Ernst, R. J.; Komor, A. C.; Barton, J. K. Biochemistry 2011, 50, 10919-10928. https://doi.org/10.1021/bi2015822

37. Geldmacher, Y.; Rubbiani, R.; Wefelmeier, P.; Prokop, A.; Ott, I.; Sheldrick, W. S. J. Organomet. Chem. 2011, 696, 1023-1031. https://doi.org/10.1016/i.jorganchem.2010.10.034

38. Siu, F. M.; Lin, I. W. S.; Yan, K.; Lok, C. N.; Low, K. H.; Leung, T. Y. C.; Lam, T. L.; Che, C. M. Chem. Sci. 2012, 3, 1785-1793. https://doi.org/10.1039/c2sc00620k

39. Dieckmann, S.; Riedel, R.; Harms, K.; Meggers, E. Eur. J. Inorg. Chem. 2012, 813-821. https://doi.org/10.1002/ejic.201101175

40. Wang, H. M. J.; Lin, I. J. B. Organometallics 1998, 17, 972-975. https://doi.org/10.1021/om9709704

41. Palomo, C.; Aizpurua, J. M.; Balentová, E.; Jimenez, A.; Oyarbide, J.; Fratila, R. M.; Miranda, J. I. Org. Lett. 2007, 9, 101-104. https://doi.org/10.1021/ol0626241

42. Isobe, T.; Fukuda, K.; Yamaguchi, K.; Seki, H.; Tokunaga, T.; Ishikawa, T. J. Org. Chem. 2000, 65, 7779-7785. 
https://doi.org/10.1021/jo000746f

43. Jo, D.; Han, S. Org. Lett. 2019, 21, 6045-6049.

https://doi.org/10.1021/acs.orglett.9b02203

This paper is an open access article distributed under the terms of the Creative Commons Attribution (CC BY) license (http://creativecommons.org/licenses/by/4.0/ 